\title{
International activities in HF sky-wave field-strength estimation (period 1956-1991)
}

\author{
Peter A. Bradley \\ Consultant, Slough, Berks SL2 3ES,U.K.
}

\begin{abstract}
Methods for the determination of the strengths of radio signals reflected from the ionosphere and propagated to distant locations are required for service planning and circuit operation. Efforts are described following World War II to arrive at agreed procedures and some of the features of the various empirical prediction methods that have been formulated over the years are discussed. The problems are highlighted of determining a «best» method from among those available. Measurement data collected for this purpose are reviewed and attention is drawn to their limitations of accuracy and coverage. Even comparison of predicted and measured values is not straightforward, and the techniques that have been developed to do this are considered.
\end{abstract}

Key words ionosphere - radio waves - high frequency - field strength - prediction

\section{Introduction}

Following the early experiments at the turn of the century by Guglielmo Marconi, firs carried out in Bologna and later in England, confirming that radio waves propagate over a distance, it soon became evident that such signals could be used to communicate information. Trials to greater and greater distances via the so-called «ground wave» culminated in 1901 in the classic experiment of reception at St. John's, Newfoundland, of signals radiated from Poldhu, Cornwall, England, a distance of 3500 $\mathrm{km}$, confirming the presence also of «skywaves» that travel via the ionosphere which acts as a mirror in the sky. But the ionosphere

Mailing address: Prof. Peter A. Bradley, Pandora, Scotland Drive, Farnham Common, Slough, Berks SL2 3ES, U.K.; e-mail: p.a.bradley@rl.ac.uk is very variable in space and time, with consequential changes to the possible propagation paths, signal intensities and the frequencies that can be supported. Besides geographical variations, there are systematic changes with timeof-day, season and epoch of the 11-year solar cycle. There are also major irregular day-to-day variations. It therefore becomes important to circuit operations to be able to predict for any occasion those frequencies that can propagate between specified pairs of terminals.

Unfortunately a large number of separate factors determine the state of the ionosphere, and even given a knowledge of what these are for any scenario, the amounts of calculation needed to determine the principal signal characteristics were prohibitive before the days of modern computers. Even today, uncertainties in ionosphere specification usually make full radio ray calculations inappropriate. Hence over the years a whole range of empirical prediction procedures has been developed. First calculations were carried out manually and to some extent those factors included in the calculations 
were governed more by available knowledge, personal preferences and resources than by true requirements. Predictions have remained empirical but later they became more complex as additional factors were taken into account. Arguably a point has now been reached where no improvement in accuracy is likely to be achieved with the introduction of additional calculations because of remaining uncertainties in ionospheric specification.

Before and during World War II each country tended to develop its own prediction methods, tailored to those radio circuits and paths for which there was an interest. Ionospheric sounding measurements were conducted and calculations produced each month in support of broadcast and point-to-point links. At the forefront in these activities were groups in U.S.A., Japan and within Europe in France, Germany, Italy and the United Kingdom. Particularly in Italy over the years prediction work has been carried out at a range of institutes, including in the first days the Centro Radioelettrico Sperimentale «G. Marconi», Roma. Latterly ionospheric sounding has been the purview of the Istituto Nazionale di Geofisica, Rome and applications to predictions for sound broadcasting have been conducted closely in conjunction with Radiotelevisione Italiana (RAI). The Italian Ministry of Telecommunication serves as a co-ordinating agency for Italian interests in international discussions on radio matters, including the assignment of experts to various international working groups both from governmental organisations and private telecommunication companies such as ITALCABLE. Whilst therefore a number of different Italian groups have been involved over the years and some continue with ionospheric research supportive of improved HF radio-wave propagation predictions, it is appropriate and timely that this special commemorative issue of Annali di Geofisica should include a paper reviewing a field in which those being honored have played important parts.

Section 2 reviews the activities of the International Telecommunication Union (ITU), responsible for co-ordinating radio usage among its member countries and their operating agencies, and for carrying out technical studies to do this in an optimum manner. It also discusses briefly the requirements for internationally accepted propagation prediction procedures. Section 3 considers early studies in field-strength predictions development within the different countries and in Sections 4-6 selected technical details of three successive internationally adopted prediction methods are described. The production of predictions for microcomputer evaluation, an important step forward at the time, is mentioned in Section 7. The establishment of data banks of co-ordinated field-strength measurements for method testing is considered in Section 8 and in Section 9 results of prediction-measurement comparisons are presented. Work leading up to a propagation prediction method developed for the 1987 World Administrative Radio Conference on high frequency broadcasting (HFBC-87) is described in Section 10.

\section{The International Telecommunication Union, its committees and working parties}

The International Telecommunication Union (ITU) is a Specialised Agency of the United Nations responsible among other matters for establishing regulations which have the force of international law governing the use of the radio spectrum. The Radio Regulations define various types of radio service (e.g., broadcasting, maritime, fixed, space), allocate different blocks of spectrum to these separate services, specify emission characteristics and establish assignment criteria and procedures for individual transmissions. Changes in the Radio Regulations are introduced from time-to-time at special conferences in response to revised requirements. In preparation for such conferences the former CCIR (International Radio Consultative Committee), a component body of the ITU until its replacement in 1993 by the Radiocommunication Sector (ITU-R) carrying out broadly similar functions, provided planning recommendations based on what are seen as good technical practices.

Hitherto CCIR procedures have involved establishing a number of Questions, Study Programmes (responsive to Questions), Reports 
of what is already known or has been determined and Recommendations (when the work is complete). The CCIR and its successor ITU-R consist of a series of Study Groups responding to particular areas and Study Group 6 (now Study Group 3) is concerned with «Propagation in Ionised Media». In the CCIR era, meetings of the Study Groups were held at roughly two-yearly intervals to update texts, and then these were endorsed at subsequent Plenary Assemblies where all interests were co-ordinated. However, to handle the more complex task the Study Groups established a number of Interim Working Parties (IWP's) to work primarily by correspondence, though these could also meet from time to time.

The New Delhi 1970 CCIR Plenary Assembly renamed all its working parties with the initial I standing for «Interim», rather than «International», as formerly. This change was introduced to signify that IWP's should have limited lifetime and be constituted to carry out specific clearly defined objectives. The fact that IWP 6/1, concerned with developing improved methods of HF sky-wave field-strength estimation, was both one of the first working parties to be set up and one of the last to be disbanded, bears testimony to the complexities of the tasks involved. This paper documents some of the highlights in its productive, if sometimes seemingly intractable pursuits.

The work of IWP $6 / 1$ has primarily involved the development and refinement of a number of different prediction models, the production of associated computer programs for their evaluation, the promotion of measured sky-wave field strengths, the assembly and validation of measurement Data Banks and the generation of procedures for comparing measurements and predictions in order to assess prediction accuracy. These different aspects are reviewed below.

\section{Early studies}

Prior to and during World War II there had been intensive efforts in a number of countries in modelling the performance of high frequency radio waves reflected from the ionosphere, and when the CCIR again became operative after hostilities ceased, propagation studies formed an important element in its activities. The need to have agreed propagation models for service planning was recognised as critical to optimum spectrum utilisation, particularly at HF where congestion was already becoming a serious problem with not all requirements being met with acceptable circuit quality. Radio users were encouraged to use these same models for the design and operation of their own radio circuits.

By the time of the VIIth Plenary Assembly (London, 1953) the work was split into Study Groups, where formerly all business had been conducted in full plenary sessions. Prominent among contributors to considerations of the preferred method of signal-strength determination were the U.S.A., where staff at the Central Radio Propagation Laboratory (CRPL) of the National Bureau of Standards had developed the method of Circular 462 (NBS, 1948) and a few years later another group at the Signal Corps had formulated the Radio Propagation Unit RPU-9 method (Laitinen and Haydon, 1950). A combined French/German team had devised the method SPIM (Service de Prévision Ionospherique Militaire) (Halley, 1965) whereas in the U.S.S.R. the method of Kasantsev (1956) was in use. In Japan a group at the Radio Research Laboratories had produced a method which took account of ground sidescatter (Miya and Kanaya, 1955). The question arose: which of these methods was most accurate? The International Frequency Registration Board (IFRB), now disbanded, was at that time the part of the ITU responsible for maintaining a Master Register of all radio emissions and carrying out technical examinations of all proposed new transmissions. The IFRB had incorporated the Circular 462 method into their Technical Standards, but there were those both in the U.S.A. and elsewhere who favoured the RPU-9 approach.

The VIIIth Plenary Assembly was held in Warsaw in 1956. Recommendation 177 was adopted calling for a study of the respective merits of the then available methods and establishing an International Working Party (IWP VI/I) under the chairmanship of D. Lepechin- 
sky (France) to «advise the IFRB on the precision of the method of RPU-9». The first participants were from France, Czechoslovakia, F.R. Germany, Japan, Romania, the U.S.A. and the U.S.S.R., together with strong and active support from both the IFRB and CCIR Secretariats. Initial studies were conducted by correspondence. The IWP first met in Geneva in December 1957.

Germany presented the method of Beckmann (1958) for consideration and the U.K. joined the group, offering the Special Report 27 method of Piggott (1959). What was needed, it was agreed, was a programme of standard measurements to establish a database against which the different predictions could be compared. Two sub-groups of the IWP were created and reported to the IXth (Los Angeles, 1959) Plenary Assembly. Sub-group A under the chairmanship of T. Gautier (U.S.A.) studied the details of the different methods and their accuracies. Sub-group B (Chairman G. Millington, U.K.) made proposals for a co-ordinated measurement programme involving a number of key measuring stations. Use of existing standard time transmissions in the U.S.A., Japan and South Africa was envisaged, with standardised receiving equipment and antennas to be deployed in several named countries. Questionnaires were prepared seeking support for the measurement programme and proposing common tabulation sheets. The CCIR Secretariat was asked to be responsible for the analysis of all the resulting pen-chart recordings that were received. Concern was expressed at the size of the job, but in the event the amount of data collected was rather minimal. The key-measuring station concept foundered with ad-hoc measurements being made only by a few groups, particularly those in Japan and Germany.

By 1965 representatives of India had joined the IWP and had offered a prediction method for tropical areas. Canada had a method for northern latitudes. Studies expanded to a consideration of seven methods. The work was aided somewhat by the fact that by then several of these methods were coded for computer evaluation. However, no unanimous conclusions were reached. The Working Party gave consideration to developing a new composite
CCIR method to satisfy the different viewpoints. There were many discussions of ways of modelling ionospheric absorption, propagation above the basic MUF, sporadic- $E$ support and off-great-circle scatter propagation. It was to be several more years though before a first CCIR method appeared, and was recommended for «interim use».

\section{The first CCIR interim prediction method (Report 252-2)}

A co-author of the RPU-9 method, G.W. Haydon, who by this time had moved to the CRPL in the U.S.A., was invited in 1964 to take over the chairmanship of IWP 6/1. Haydon joined a group there that included W.B. Jones, R. Gallet and M. Leftin who had developed a method of numerical mapping to represent the geographical and temporal variations of ionospheric characteristics (Jones et al., 1969). He worked with D.L. Lucas and produced both an expanded manual field-strength prediction method involving lots of charts and nomograms (Haydon and Lucas, 1966), and then later a fully computerised method (ITSA-1) relying on the numerical maps (Lucas and Haydon, 1966). The IWP strived to produce both an agreed computer-based method of evaluation and also a simpler manual method, but by 1968 they stated their disinterest in the manual method because of the amounts of time involved in its implementation, to say nothing of the strong likelihood of numerical error in the process. (It was though many years later before the CCIR finally deleted from its texts the aim of generating manual methods).

The IWP concluded that there really were no major differences in the methods considered, merely varying approaches to modelling the separate factors that were agreed as important. A «typical» method, ITSA-1, was circulated to see «if it could be used as the basis of an international method and to determine what modifications would be required before such a method would be acceptable». The numerical maps of ionospheric characteristics which had been embodied into Report 340 and recom- 
mended for use by the Oslo 1966 Plenary Assembly (Recommendation 434, now Recommendation ITU-R P.1239) were to be employed (fig. 1). A mode treatment was to be followed with ray-path angles determined in accordance with geometrical considerations as formulated by K. Rawer (Germany). Spatial attenuation would be based on ray-path distance. Ionospheric absorption would be estimated in terms of solar index and either solar-zenith angle or the ionospheric characteristic $f_{0} E$ as proposed by A.N. Kasantsev (U.S.S.R.). The absorption winter anomaly and additional absorption at auroral latitudes would be incorporated. Nighttime absorption would be determined as suggetsted by N. Wakai (Japan). Ground-reflection losses and day-to-day variations of signal strength would also be modelled. For later consideration in an improved method would be the role of the $F_{1}$-layer, deviative absorption, above-the-MUF loss propagation, sporadic- $E$ modes and off-great-circle propagation. Until this new method was formulated, further measurement campaigns and prediction comparisons were abandoned.

A special 3-day meeting in September 1969 led to the Working Party submitting to the Study Group for adoption a «provisional interim» method. In the event this method followed closely the ITSA-1 formulations. Raypath equations ignored the Earth's magnetic field and were appropriate to propagation via parabolic $E$ and $F_{2}$-layers. Absorption was expressed in terms of a modified solar-zenith angle. Basic MUF and signal strength followed a chi-squared law of day-to-day variation. An important element was an empirical «excess lossfactor» designated $Y_{p}$ to normalise predicted

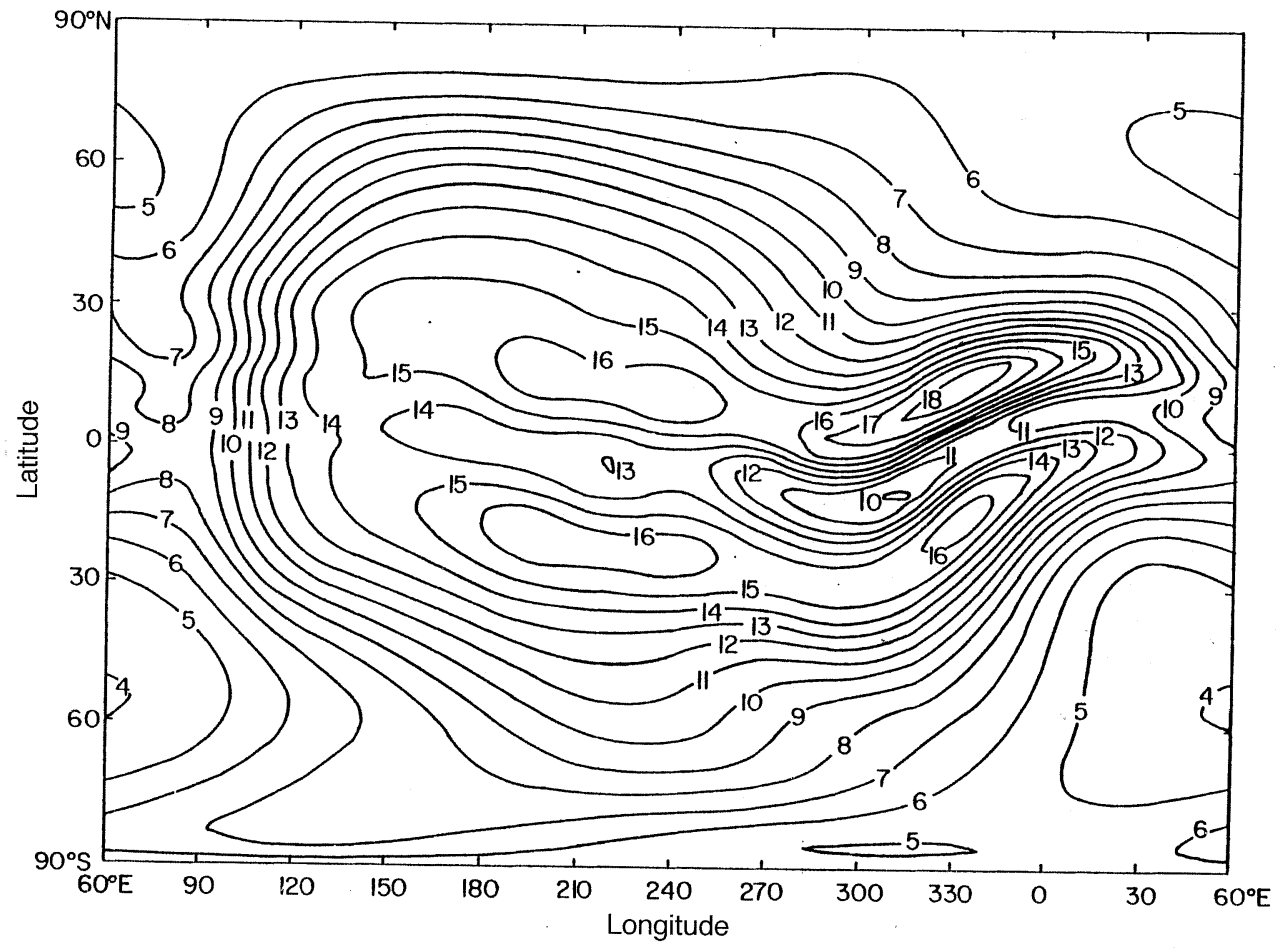

Fig. 1. World map of the ionospheric characteristic $f_{0} F_{2}$, related to the maximum ionisation density in the $F_{2}$ region of the ionosphere, for 00h UT in March at high solar epoch, as used in the first CCIR HF prediction
method. 
values to median figures given by the measurement data set which had been used in formulating the RPU-9 procedure. $Y_{p}$ was taken as a variable with time-of-day, season and latitude and to be different for path lengths less than or more than $2500 \mathrm{~km}$.

In its report, the IWP stated that «adoption by the CCIR at this time is urged primarily for two reasons: 1) it will provide organisations and administrations with an interim method for their common use, and 2) it will provide a point of departure with which the administrations may compare their prediction methods and measurement results and offer specific documentation for the improvement of the method». The Study Group accepted the proposal and the New Delhi (1970) Plenary Assembly adopted the method of Report 252-2. The U.S.A. administration then furnished the associated computer program. It was though a further eight years until the 1978 Kyoto Plenary before Recommendation 533 was adopted advocating use of the method provisionally, until the computer program for the second method that had by then been generated, was complete.

\section{The second CCIR interim prediction method (Supplement to Report 252-2)}

Following evaluations of the first method by several administrations, the IWP went on to study possible improvements. Plans were drawn up to revise the expressions for daytime and night-time absorption, to introduce an allowance for polarisation-coupling loss, to consider the extension to frequencies above the basic MUF as included in the Beckmann method and to take account of ionospheric focusing. The need was recognised to find a way of being able to reduce the excess-system loss «fiddle-factor» values which are claimed to include composite allowances for propagation effects not otherwise explicitly taken into account.

With the present author appointed chairman, studies continued along lines already established. There was clear agreement on several items, but with other topics different possible approaches emerged. A new package proposal was put together and debated at a Geneva meeting in February 1976. Perhaps not surprisingly this included as a kernel many elements that were in current use within the United Kingdom. The CCIR numerical maps of ionospheric characteristics were retained. Ray-path analyses were applied for the model ionosphere of Bradley and Dudeney (1973), consisting of parabolic $E$ and $F_{2}$-layer segments together with a linear $F_{1}$-layer segment for greater realism (fig. 2). The ionosphere was separately generated for each hop and a homing procedure applied to find the elevation angles corresponding to a given ground range. The George and Bradley (1974) ionospheric absorption formulation based on an analysis of measured vertical-incidence absorption data together with an obliquity factor transformation was included. Horizon and antipodal focusing were allowed for. Explicit expressions for polarisation-coupling loss for single-hop modes were added and the $Y_{p}$ concept was not used. For the estimation of auroral absorption, the IWP decided to embody new formulae of Foppiano (1975) derived from a study of riometer data, rather than to use values determined by comparing $Y_{p}$ for auroral and non-auroral paths. Sporadic- $E$ reflection losses were determined by means of expres-

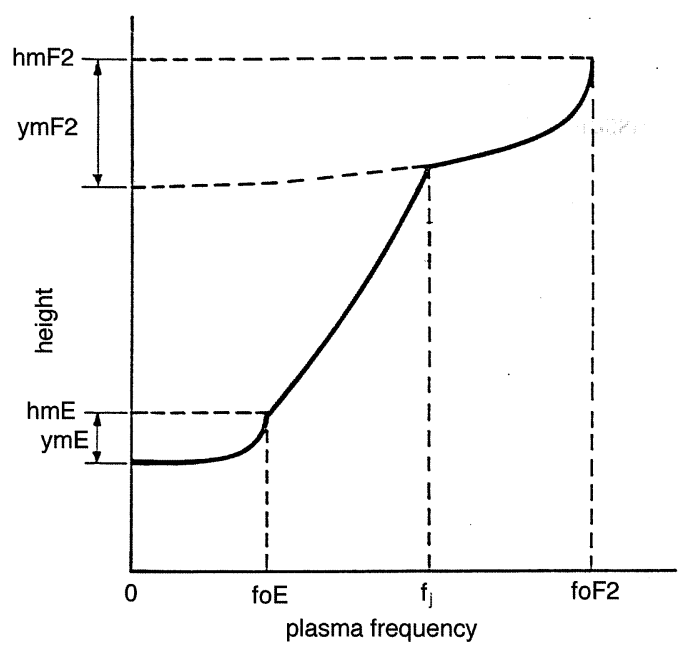

Fig. 2. Height distribution of electron density given by the model of Bradley and Dudeney (1973). 
sions generated within another Working Party, IWP 6/8, established to investigate such effects. For the estimation of above-the-MUF loss a simple formula was fitted to measured data collected in the U.S.A. by Wheeler (1966), giving a parabolic increase of decibel loss with frequency-to-basic MUF ratio.

The Kyoto (1978) Plenary Assembly adopted this new method, but because of some concerns for its complexity, coupled with the fact that the associated computer program was not yet available, it was designated as the method of the Supplement to Report 252-2, with the first method also retained. Completion of the computer program proved a lengthy affair in which groups in several countries as well as the IFRB and CCIR Secretariats assisted over a number of years. A meeting of the IWP in Warsaw in 1980 gave particular emphasis to plans for program completion. By 1982 the program was deemed to be complete, though there were still minor changes being effected up to December 1985 when it became available for purchase. Extensive documentation followed only in March 1987. The Geneva (1982) and Dubrovnik (1986) Plenary Assemblies continued to recommend use of the first method until the second method had been further tested. Meanwhile a much simpler method had been developed by IWP $6 / 12$ for use in broadcast planning (see below). Tests against observational data showed this latest method to be not substantially less accurate and so at the Dusseldorf (1990) Plenary Assembly Recommendation 533-2 advocated this third method for general use, but with the first and second methods retained for «specific cases».

\section{Further predictions development and the method of CCIR Report 894}

The ITU Administrative Council scheduled the first session of a World Administrative Radio Conference on High Frequency Broadcasting (HFBC) in January 1984, and in preparation for that conference CCIR Study Group 6 was charged by the 1979 (Geneva) General World Administrative Radio Conference with making recommendations concerning the propagation method to be used. IWP 6/12 was established in June 1980 under the chairmanship of D.L. Lucas (U.S.A.) with this remit. Membership included many participants from within IWP $6 / 1$, and so because of the urgency of the broadcast studies, other matters were pursued less vigorously. Nonetheless IWP $6 / 1$ continued to review aspects of predictions development and testing and thereby to assist IWP $6 / 12$ as required, particularly in making measurement data available.

With the existing computers of the day, the broadcast planning requirement was seen as dictating a relatively simple approach in which it was essential that the large number of computations needed in compatibility investigations could be carried out in a relatively short time, even if this were to involve some loss of accuracy. IWP $6 / 12$ held three separate meetings between January 1981 and February 1983 with active Italian participation. It reviewed some twenty different methods submitted to it. The methods of Report 252-2 and Supplement were soon discarded as too complex for the task in hand. Eventually it was determined to formulate a new hybrid method consisting of a rayhop treatment for path ranges up to $7000 \mathrm{~km}$, composite mode empirical expressions derived from the fit to measured data beyond $9000 \mathrm{~km}$ and linearly interpolated values over the $7000-9000 \mathrm{~km}$ transition region. Up to $7000 \mathrm{~km}$ the technique proposed by the U.S.A. followed somewhat similar procedures to those of Report 252-2, but with simpler critical frequency and basic MUF expressions. Ray paths were those appropriate to reflection heights given only in terms of $M(3000) F_{2}$ independent of path length and frequency. The above-theMUF loss formulation of the Supplement was introduced. Auroral absorption loss was adduced from the Report 252-2 excess-system loss tables. Beyond $9000 \mathrm{~km}$ the Beckmann method was adopted, which is based on an empirical fit to measured data collected in Germany, with field strengths given in terms of transmitter power, path length and a function which depends on wave frequency, and estimates of the maximum frequency that can propagate via ionospheric refraction alone and the somewhat greater maximum frequency provid- 
ing satisfactory system performance. Different amplitude standardisation factors were incorporated for both the short and long-path components of the method, determined from comparisons with the IWP 6/1 Data Bank of measurements (see later). This composite method was adopted by the Geneva (1982) CCIR Plenary Meeting as the method of Report 894.

Its task completed, IWP 6/12 was disbanded. Since that time IWP $6 / 1$ reviewed many items of predictions development. It monitored activities elsewhere and continued to study various aspects of ionospheric mapping and modelling and the determination of propagation effects. The respective merits were compared of grid-point storage, Jones-Gallet basis functions (Jones et al., 1969) and simpler empirical relationships (Damboldt and Süßmann, 1986; Levine et al., 1978) for representing ionospheric data (Banks et al., 1983). Better ways of determining ionospheric absorption (Peres, 1990) and auroral absorption (Zhulina et al., 1984) were addressed. Approaches to circuit reliability estimation were considered (Maslin, 1978). Responses to a survey questionnaire among radio users on requirements for propagation predictions were collated (Bradley and Vernon, 1984). Operational mainframe prediction methods in use within Argentina, Australia, China and India were examined (CCIR, 1978; Lakshmi et al., 1987; Turner, 1985).

In the light of the results of the accuracy tests for the Report 894 method in comparison with the more complex methods and noting the delays in subsequent sessions of the Broadcasting Conference, it was deemed inopportune at that stage to try to develop a radically new prediction method. Instead, efforts were devoted to improving the Report 894 method accuracy and in seeking to enhance its applicability. IWP 6/1 was instrumental in introducing a number of changes to that method which were approved by the 1996 (Dubrovnik) and 1990 (Dusseldorf) Plenary Assemblies, including those to allow for the range dependence of mirroring height and to better model antipodal focusing and the method of distance interpolation on 7000-9000 $\mathrm{km}$ paths. The procedure was extended to include the estimation of signal/noise ratio Lowest Usable Frequency (LUF) and circuit reli- ability. Expressions for available signal powers were formulated which include summing the separate mode components, also taking account of receiving antenna gain. Interactions were maintained with IWP 10/1 tasked to develop an agreed set of gain expressions for the range of antennas in common use (CCIR, 1988; Gibson, 1989). A meeting of IWP 10/1 hosted by Vatican Radio was held in Rome in February 1991. Italian contributions included comparisons of theoretical and measured antenna radiation patterns. Mainframe and microcomputer programs for the latest version of the Report 894 method were developed, tested internationally, documented and forwarded to the CCIR Secretariat for distribution and sale.

\section{Microcomputer predictions}

The rapid proliferation of microcomputers in the first half of the 1980's led to the evolution of a number of different propagation prediction procedures for microcomputer evaluation. Such procedures were developed both by commercial organisations and in a significant number of cases by radio amateurs and other enthusiasts. One method was produced in Italy by RAI staff. Each designer of a procedure chose to include those aspects he considered to be important and, although many had common elements, probably no two were completely alike. The first procedures to be evolved were simplifications of mainframe methods, but as storage capacities and computing speeds increased, so did method complexities. Report 1013 gave a specification devised by IWP 6/1 for a proposed CCIR microcomputer method.

The IWP met in Lannion, France in 1986 to review available methods. Several groups brought their equipment and programs and demonstrated these. A set of standard comparison circuits and operating conditions was specified. A questionnaire was formulated seeking details of available methods together with sample results, and this was distributed to more than fifty groups worldwide known to be active in the area. Responses were good and a survey report was issued presenting replies (Bradley and Vernon, 1988). There was concern though 
that it would be impossible to continue to maintain awareness of all methods being produced, and by the time of a subsequent Working Party meeting in Brasilia in 1988 the conclusion was reached that it would be feasible to have a microcomputer version matching without significant simplification the mainframe version of the Report 894 method. This view was accepted by the Study Group and the IWP proposal to delete Report 1013 was adopted.

Groups in France and the U.K., active within the IWP, had each developed microcomputer procedures closely following the Report 894 method. So the decision was made to combine these. A series of different graphical and tabular outputs were provided. A cursor-interactive input arrangement included adequate «help» instructions and was versatile in allowing many inter-related calculations with a minimum of separate input data. This well-documented program (REP894) was available on floppy disk from the CCIR Secretariat, together with other disks containing the associated ionospheric reference data needed for its evaluation. The IWP also furnished the Secretariat with a microcomputer program MUFFY for the estimation of maximum and best frequencies that can propagate over point-to-point links. This followed the procedures of Part 12 of Report 340 (as now embodied in Recommendation ITU-R P.1240).

\section{Signal-strength measurements and Data Banks}

Early studies of the accuracy of contending prediction procedures used different measurement data sets. Comparative results were therefore inconclusive and the need for an independently agreed data set was evident. When in 1977 attention returned to the establishment of a data bank of measurements, initially in order to test the relative accuracies of the methods of Report 252-2 and Supplement, only two groups were systematically making signal-strength measurements. Standard time transmissions from U.S.A. and Japan were being monitored by the Japanese Radio Research Laboratories and the strengths of signals from a number of different transmitters, mainly those radiating meteorological information, were recorded by the Fernemeldetechnische Zentralamt (now part of Deutsche Telekom), Germany. Under IWP $6 / 1$ initiatives these data were subjected to intensive scrutiny regarding the calibration factors that were applied, and a method of data normalisation was evolved to give equivalent sky-wave field strengths and available receiver powers for $1 \mathrm{~kW}$ isotropic transmitter radiation.

A plan of campaign for predictions comparisons was evolved, and because of differences in amounts of data it was agreed to formulate a data set (later designated Data Bank A) consisting of monthly median values for the even hours for just four months of the year and only for a limited selection of years for each circuit. Later, other groups furnished data, notable amongst these being All India Radio and the European Broadcasting Union (EBU), providing information for a range of broadcast circuits. A second smaller data set (Data Bank B) was assembled in June 1982 and subsequently this was combined with the first data set to give Data Bank C. Efforts were made to stimulate further data collection initiatives through appropriate CCIR Resolutions. In conjunction with the U.K. administration, a completely revised Report 253 was produced discussing how measurement data should be collected, tabulated, analysed and forwarded to the Secretariat. Subjects addressed specifically within the IWP included the minimum number of days in a month that measurements need to be collected in order to provide meaningful results, data tabulation arrangements, and how to normalise the measurements to corresponding sky-wave field strengths so as to be compatible with predicted parameters. Lengthy debates one evening in sessions at an IWP meeting in Abingdon, U.K. in 1985 led to a simplified algorithm for elevation-angle determination needed to allow for antenna gain in the data normalisation process, but with which some participants still have misgivings. Rules were devised for computing median values when dealing with incomplete data sets. In March 1986 a new Data Bank D was created including all measurements that passed the agreed scrutinies with regard to likely accuracies and sampling procedures. It embodied 
the data from Data Bank C, together with some additional results. Normalisations had been applied as agreed in Abingdon. Data Bank D consists of more than 16000 monthly median hourly measurements for 181 circuits with frequencies ranging from $2.5 \mathrm{MHz}$ upwards. A slightly corrected version, Data Bank D1 issued in January 1989 remains as the definitive set for use (Süßmann, 1989).

Many IWP 6/1 participants, including those from Italy, also took part in the activities of IWP 6/14 (Chairman Th. Damboldt, Germany), established by Study Group 6 in 1987 to make recommendations for a new coordinated international programme of field-strength measurements. These data were needed, it was argued, in order to improve the propagation model to be used by the high frequency broadcasting conference. A Vienna meeting of IWP 6/14 in October 1987 led to proposals for an ambitious network consisting of 9 globally distributed dedicated transmitters radiating coded information with any number of remotely operated and micro computer-controlled receivers recording data on floppy disk (Report 1149). Regrettably, despite the repeated efforts of Director CCIR to gain the support of administrations for this campaign, the necessary resources were not forthcoming to allow it to be introduced, though transmissions from Melbourne, Australia and Stavanger, Norway were initiated.

The 1989 Study Group 6 Final Meeting transferred further data collection studies to new IWP 6/15 (Chairman N. Wakai, Japan). Close relations continued with IWP $6 / 1$ and in December 1990 a joint Geneva session was held. It was agreed there were outstanding problems still to address regarding data normalisation, the merits of having a new data bank with all values expressed in available receiver power and whether it is desirable and feasible to have a data bank containing daily values. These matters were considered in later years via new groups. The particular advantages of having signal measurements expressed as equivalent available receiver powers has been recognised in that thereby inconsistencies are avoided arising from differences in antenna gain allowances in the predictions and with the measurement normalisations. Some progress has been made in recent years towards the compilation of a new data bank with values expressed in available receiver power.

\section{Prediction-measurement comparisons}

Throughout its lifetime IWP 6/1 made many studies involving comparisons of predicted and measured field strengths and contributed results for inclusion in Report 571 summarising such information. It became evident during this work that there are a number of problems in making deductions from such comparisons not a straightforward matter. When there is a measurement and a prediction, a difference can be determined. But what if one or the other is missing? Is that case always ignored from the sample? Are we to have different sample sizes when comparing separate predictions with the same data set? What parameter should be used as a figure-of-merit of a given prediction procedure? Some methods include a normalisation factor to eliminate the median error, so should the r.m.s. difference regarded as indicative of the spread of results be what counts? Are predicted median values those appropriate to all days of the month, or only those that propagation is deemed possible? What happens when the fractions of days predicted and measured differ? At this time not all such questions are still completely resolved.

Attempts have been made to formulate comparison procedures in terms of daily measured values, but these are not yet fully evolved and currently there are no agreed measurement data sets to use. At the Brasilia (1988) meeting IWP $6 / 1$ proposed a standard procedure for prediction-measurement comparisons using monthly median data with results shown grouped separately in various ways as a function of path length, latitude, frequency, time etc. This was adopted as Report 1150 and all existing CCIR recommended field-strength prediction methods including that proposed to the HFBC have been evaluated and tested in this way (Süßmann, 1988). Examples of the types of comparison statistics thereby produced are shown in table I. The technique remains current within Recommendation ITU-R P.1148-1. 


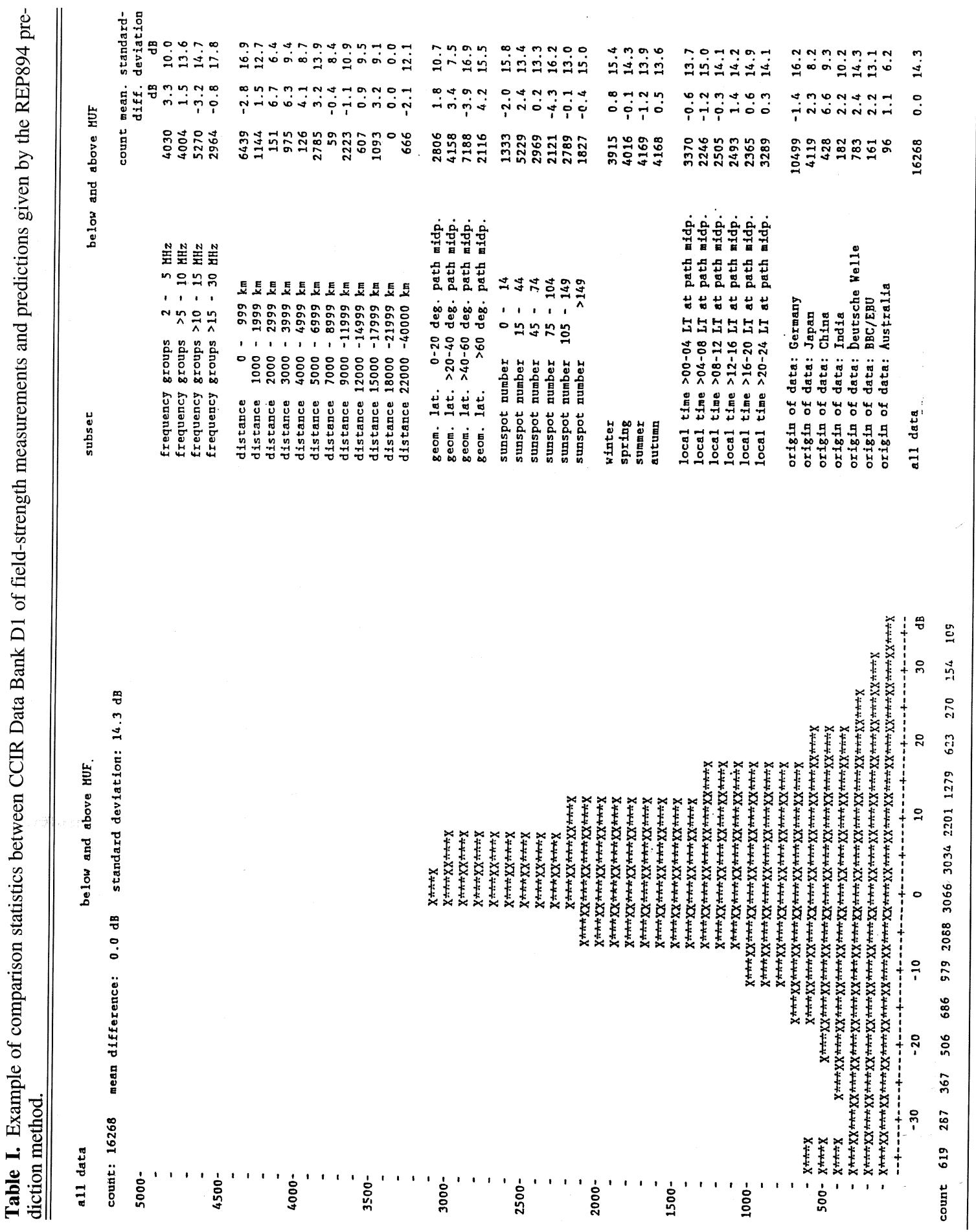




\section{Review of the propagation method adopted by the 1987 HF Broadcasting Conference (HFBC-87)}

HFBC-87 in its Recommendation 514 invited the CCIR to study and if necessary to make recommendations for improvements in its method of modelling propagation effects. This task was assigned by Study Group 6 to IWP 6/1 with the remit that a first report be prepared for approval by the 1990 Plenary Assembly and a second and final report be made available 17-months before the scheduled follow-on 1993 Conference. The resulting revised HFBC method is a development of the method proposed in 1981 by IWP 6/12. Since too the CCIR version on which it is based had undergone two rounds of revision, there then became important differences between the two methods.

IWP 6/1 met in London in January 1990 having perused carefully the associated computer codes, catalogued the areas of commonality between the two methods and highlighted the differences (CCIR, 1990). It noted which of these differences in its opinion were important and which not. It made three specific recommendations for change in the HFBC method: i) the possibility of screening of $F_{2}$-layer reflected modes by the $E$-layer should be tested for path lengths up to $9000 \mathrm{~km}$; ii) the formula for antipodal focusing should be changed to one more consistent with observational data; and iii) logarithmic as opposed to linear interpolation of field strength should be applied between the short and long-path predicted values over the 7000-9000 km distance range.

Certain aspects were noted as requiring further study and these were considered at length at a final meeting of IWP $6 / 1$ held at Kleinheubach, Germany in May 1991 (see Plate). New methods of determining ray-path elevation angles, locations of control points, and several different approaches to estimating the additional losses that arise for frequencies above the basic MUF, for which it is believed propagation is sustained by ionospheric scatter and ground sidescatter, were addressed at length. The second CCIR report to the 1993 conference (CCIR, 1991) includes a full specification of the recommended HFBC method and provides then latest available values of the standardisation factors $L_{z}$ and $L_{y}$, which are the empirical correction terms embodied in the method so as to eliminate median differences when compared with Data Bank D1.

\section{Concluding remarks}

IWP 6/1 had only three chairmen over its lifetime: D. Lepechinsky (France; 1956-1964), G.W. Haydon (U.S.A.; 1964-1975) and P.A. Bradley (U.K.; 1975-1991). Over the years there was active and sustained participation from representatives of 17 countries and five private operating agencies, as well as stalwart support from both the CCIR and IFRB Secretariats. Overall, more than 350 documents were generated - nearly a $2 \mathrm{~m}$ wide library. As well as work conducted by correspondence there had been more than twenty specifically scheduled meetings together with other informal discussion sessions. Several individuals personally contributed to the work for over a decade.

Nonetheless, when the Working Party was disbanded in 1991 with the re-organisation of CCIR committees there remained significant studies that should be pursued further, even if many of these had been with the IWP since its inception. The importance of scatter propagation, whether from the ionosphere or ground, needed more consideration. Then available predictions were sorely deficient in not properly allowing for receiving antenna gain and the enhancement of existing computer codes with an approved library of antenna gain routines had to be seen as a priority. More work was needed to refine the models of ionospheric absorption, particularly for the higher latitudes. Resolution was wanted of the respective merits of different methods of reliability determination. Other matters too needed to be addressed.

Following the revision of CCIR working practises, studies formerly conducted within IWP's were transferred to new Working Parties. Working Party 6A, also chaired by P.A. Bradley, made progress along some of the above lines. In particular, yet a further new field-strength prediction method (Recommendation 533, currently Recommendation ITU-R 




Members of IWP 6/1 at their 1991 final meeting at Kleinheubach, a Schloss (Castle) situated on the banks of the River Main which is a tributary of the Rhine in Germany. Kleinheubach Schloss is a study centre of Deutsche Telekom. In the picture (from left to right) are Noboru Wakai (Japan), John Wood (USA), Mike Dick (U.K.), George Lane (U.S.A.), Kevin Hughes (CCIR Secretariat), Peter Süßmann (Germany), Peter Bradley (Chairman U.K.), Thomas Damboldt (Germany), Angela Vernon (U.K.), Les Barclay (Chairman Study Group 6, U.K.), Don Ross (Canada), Richard Davis (U.K.), Jurgen Hortenbach (Germany), Mashhour Zamanian (Iran), Rudi Hanbaba (France), Rodoljub Medan (Yugoslavia), Ian Davey (U.K.), Alberto Torres-Marin (Spain), and Ayumu Ohta (Norway).

P.533-5) was generated as a development from the Report 894 method. A new Recommendation was formulated for reliability determination. This is taken as the probability that a required signal/noise ratio will be attained and is given in current Recommendation ITU-R P.842-1. Ongoing groups examined improved methods of estimating ionospheric absorption and above-the basic MUF losses. The collection of additional measurement data and their standardisation remain important topics (Recommendation ITU-R P.845-3). In 1994 Working Party 6A became Working Party 3L with the same participants, and from January 1997 the tasks of HF predictions improvement has continued under the chairmanship of $R$. Hanbaba (France). A companion paper describes more recent developments.

\section{Acknowledgements}

Acknowledgement is made to Mr. Alfredo Magenta of Radiotelevisione Italiana (RAI), International Chairman of Study Group 10 (Sound Broadcasting) of the Radiocommuni- 
cation Sector of the International Telecommunication Union, for the provision of information describing Italian activities in the above fields of study. Thanks are extended to Professor N. Wakai, formerly of Tokai University, Japan and to Mr. G. Lane, formerly of Voice of America, U.S.A., for the provision of the Plate photograph.

\section{REFERENCES}

BANKS, P.H.T., B.H. MiLleR, M.I. DiCK and P.A. BRADLEY (1983): Storage of the CCIR reference maps of ionospheric characteristics for microcomputer evaluation, CCIR IWP 6/1 Doc. 244.

BeCKMANN, B. (1958): Über Beziehungen der Felstärke $\mathrm{zu}$ den Grenzen des Übertragungsfrequenzbereiches (LUF-MUF), (Concerning the relation of the field intensity to the limits of the transmission frequency range (LUF-MUF)), NTZ, 11, 523-528.

BRADLEY, P.A. and J.R. DUDENEY (1973): A simple model of the vertical distribution of electron concentration in the ionosphere, J. Atmos. Terr. Phys., 35, 2131-2146.

BradLeY, P.A. and Mrs. A. VERNON (1984): Requirements for ionospheric propagation predictions, CCIR IWP $6 / 1$ Doc. 248.

BradLEy, P.A. and Mrs. A. VERnON (1988): Available microcomputer-based $\mathrm{HF}$ propagation prediction procedures, CCIR IWP 6/1 Doc. 320.

CCIR (1978): HF prediction procedures used in India, China and Argentina, CCIR IWP 6/1 Doc. 308.

CCIR (1988): Study Group 6 requirements for antenna-gain pattern information, CCIR IWP 6/1 Doc. 330.

CCIR (1990): Improvements in the propagation prediction method to be used for the HF bands allocated exclusively to the Broadcasting service (first CCIR report to HFBC-93), CCIR IWP 6/1 Doc. 359.

CCIR (1991): Improvements in the propagation prediction method to be used for the HF bands allocated exclusively to the Broadcasting service (second CCIR report to HFBC-93), CCIR IWP 6/1 Doc. 393.

DAMBOLDT, TH. and P. SÜBMANN (1986): A simple method of estimating $f_{0} F_{2}$ and $M(3000)$ with the aid of a home computer, CCIR IWP 6/1 Doc. 273.

FoPPIANO, A.J. (1975): A new method for predicting the auroral absorption of HF sky waves, CCIR IWP $6 / 1$ Docs. 3 and 10.

George, P.L. and P.A. BRADLEY (1974): A new method of predicting the ionospheric absorption of high frequency waves at oblique incidence, Telecommun. J., 41 (5), 307-312.

GIBSON, A.J. (1989): Requirements of IWP 6/1 for an antenna-gain package for microcomputer-based HF propagation assessments, CCIR IWP 6/1 Doc. 332.

HALlEy, R. (1965): Méthode de calcul des prévisions de point à point aux distances comprises entre 2500 et 10500 km, Centre National d'Etudes des Télecom- munications, Division des Prèvisions Ionosphèriques, France.

HAYDON, G.W. and D.L. LuCAS (1966): Technical considerations in the selection of optimum frequencies for high frequency sky-wave communication services, ESSA Monograph.

Jones, W.B., R.P. GRAHAM and M. LEFTIN (1969): Advances in ionospheric mapping by numerical methods, U.S. Government Printing Office, Washington DC 20402, ESSA Tech Report ERL 107-ITS 75.

KASANTSEV, A.N. (1956): Developing a method of calculating the electrical field strength of short radio waves, in Trudy IRE, Transactions of the Institute of Radio Engineering and Electronics of the Academy of Sciences, USSR, 2, 134.

LAITINEN, P.O. and G.W. HAYDON (1950): Analysis and prediction of sky-wave field strength intensities in the high frequency band, U.S. Army Signal Radio Propagation Agency, Ft. Monmouth, NJ., Technical Report 9.

LAKSHMI, D.R., B.M. RedDy, S. ShastRI and A.B. GHOSH (1987): Computerized HF link at NPL, India, CCIR IWP 6/1 Doc. 288.

Levine, P.H., R.B. Rose and J.N. MARTIN (1978): MINIMUF-3: a simplified hf MUF prediction algorithm, in IEE Antennas and Propagation Conference Proceedings, 169, part 2, 161-167.

LuCAS, D.L. and G.W. HAYDON (1966): Predicting statistical performance indexes for high frequency ionospheric telecommunications systems, U.S. Government Printing Office, Washington DC 20402, ESSA Tech. Report IER 1-ITSA 1.

MASLIN, N.M. (1978): The calculation of circuit reliability when a number of propagation modes are present, CCIR IWP 6/1 Doc. 79.

MIYA, K. and S. KANAYA (1955): Radio propagation prediction considering scattering wave on the Earth's surface, Rep. Ion. Res. Japan, 9 (1), 1-15.

NBS (1948): Ionospheric radio propagation, National Bureau of Standards, Circular 462.

PERES, M. (1990): Assessment of the George-Bradley method to estimate the ionospheric absorption, CCIR IWP 6/1 Doc. 352.

PIGGoTT, W.R. (1959): The calculation of the median sky wave field strength in tropical regions, Radio Research Special Report No. 27, HMSO, London.

SÜBMANN, P. (1988): Results of applying the standardised procedure for comparing HF predictions and measurements, CCIR IWP 6/1 Doc. 324 (Corrigendum 1988) (Rev. 1 1989) (Addendum to Rev. 1 1989) (Rev. 1 Corrigendum 1 1989) (Rev. 1 Addendum 1 1989).

SüßMANN, P. (1989): Data Bank D1, CCIR IWP 6/1 Doc. 331 .

TURNER, J.F. (1985): A fast method for computing HF sky wave field strength based on the Second CCIR Interim Method, CCIR IWP 6/1 Doc. 257.

WHEELER, J.L. (1966) Transmission loss for ionospheric propagation above the standard MUF, Radio Sci., 1 (11), 1303-1308.

ZHULINA, E.M., P.V. KISHCHA, V.M. LUKASHKIN and A.V. SHIROCHKOV (1984): Additional energy losses in high-latitude radio links, CCIR IWP 6/1 Doc. 247. 